\title{
REPERCUSSÕES RESPIRATÓRIAS E HEMODINÂMICAS DO PNEUMOPERITÔNIO NA LESÃO DO DIAFRAGMA - ESTUDO EXPERIMENTAL
}

\author{
Sílvia Cristine Soldá*, francisco Cesar Martins Rodrigues, Samir Rasslan, Jacqueline Arantes G. Perlingeiro, luis Francisco Poli de
} Figueiredo, Maurício Rocha e Silva.

Trabalho idealizado pelo Serviço de Emergência da Faculdade de Ciências Médicas da Santa Casa de São Paulo e realizado na Divisão de Experimentação do Instituto do Coração/Departamento de Cardiopneumologia da Faculdade de Medicina da Universidade de São Paulo

\section{*Correspondência}

Av. República do Líbano, 2105

São Paulo/SP

Cep: $04502-100$

Tel: (11) 2176-7264

ssolda@terra.com.br

\begin{abstract}
RESUMO
OBjetrvo. Analisar as alterações hemodinâmicas e respiratórias secundárias à laparoscopia em modelo experimental de lesão diafragmática.

Métodos. Foram utilizados 15 suínos, distribuídos em três grupos com cinco animais cada, assim constituídos: Grupo I - Portadores de lesão diafragmática e avaliados sem pneumoperitônio (CLSP); Grupo 2 - Sem lesão diafragmática e avaliados com pneumoperitônio (SLCP); Grupo 3 - Portadores de lesão diafragmática e avaliados com pneumoperitônio (CLCP). Foram avaliadas variáveis gasométricas, hemodinâmicas e respiratórias.

REsultados. Houve alterações significantes da pressão média de artéria pulmonar (PAP), pressão de capilar pulmonar (PCP), $\mathrm{PAO}_{2}, \mathrm{SAO}_{2}$ e $\mathrm{SVO}_{2}$.

Conciusão. A videolaparoscopia na presença de lesão do diafragma não acarreta alterações do débito cardíaco e pode ser realizada com segurança, desde que a pressão de pneumoperitônio não ultrapasse valores de $15 \mathrm{mmHg}$.
\end{abstract}

Unitermos: Diafragma. Laparoscopia. Pneumoperitônio.

\section{INTRODUÇÃO}

A transição tóraco-abdominal é uma região delimitada abaixo do quarto espaço intercostal na fase anterior, sexto espaço intercostal na face lateral do tórax e ponta da escápula ou oitavo espaço na região dorsal, tendo como limite inferior a região epigástrica!.

Quando se analisam os ferimentos por arma branca desta região, um fato preocupante é a lesão do diafragma, pois apesar dos avanços nos métodos diagnósticos, ainda hoje o índice de lesões despercebidas é significativo ${ }^{6-8}$, com números de morbimortalidade que variam de $7 \%$ a $48 \%^{2-5}$, especialmente quando o ferimento diafragmático é isolado e não traduzido por sinais clínicos?.

Uma série de métodos diagnósticos foi proposta na literatura, como radiografia do tórax, introdução de contraste na cavidade peritoneal, lavagem peritoneal, ultra-sonografia e tomografia computadorizada, todos com elevados índices de falso-negativo ${ }^{10-15}$.

Neste sentido, a videolaparoscopia tem sido indicada de forma sistemática nestes ferimentos, para os doentes assintomáticos, a fim de descartar a lesão diafragmática, pois é consenso que possibilita uma avaliação segura do diafragma ${ }^{16-19}$.

Entretanto, existe a possibilidade de ocorrer pneumotórax durante a realização do pneumoperitônio com insuflação de $\mathrm{CO}_{2}$, pela passagem de ar para o tórax através do orifício diafragmático ${ }^{20-22^{2}}$, como relatado por Ivatury et al., em 1992!. Além disso, é possível que possam ocorrer alterações hemodinâmicas e respiratórias decorrentes do pneumoperitônio, embora não haja publicações na literatura referentes a esta situação.
O objetivo deste estudo é analisar as alterações hemodinâmicas e respiratórias secundárias ao pneumoperitônio, necessário para a videolaparoscopia, como método diagnóstico no trauma penetrante, em modelo experimental de lesão diafragmática.

\section{MÉtodos}

O experimento foi realizado na Divisão de Experimentação do Instituto do Coração/Departamento de Cardiopneumologia da Faculdade de Medicina da Universidade de São Paulo e previamente aprovado pelo Comitê de Ética em Pesquisa desta Instituição.

Foram utilizados 15 suínos da raça Landrace, fêmeas, com peso que variou de $18,5 \mathrm{~kg}$. a $27,7 \mathrm{~kg}$. provenientes da Faculdade de Medicina Veterinária e Zootecnia da Universidade de São Paulo Campus de Pirassununga e alojados no Instituto de Ciências Biológicas II da Universidade de São Paulo, alimentados com ração padronizada para a idade.

Os animais foram transportados em gaiolas individuais no dia do experimento, recebendo medicação pré-anestésica pela via intramuscular: Acepromazina a 0,2\%, 0,1 mg/ $/ \mathrm{kg}$ associada ao Midazolam, 0,75 mg/kg/. Após dez minutos foi aplicada Atropina, $0,04 \mathrm{mg} / \mathrm{kg}$.

Após um período de 20 minutos, os animais foram pesados e conduzidos à sala de experimentação.

Foram distribuídos de forma aleatória, em três grupos, com cinco animais cada, assim constituídos: 
Grupo | - Submetidos à lesão diafragmática por via laparoscópica e avaliados sem pneumoperitônio (CLSP).

Grupo 2 - Sem lesão diafragmática e avaliados com pneumoperitônio. Este foi considerado o grupo controle (SLCP).

Grupo 3 - Submetidos à lesão diafragmática por via laparoscópica e avaliados com pneumoperitônio (CLCP).

\section{Técnica operatória}

Foi obtido um acesso venoso com Abbocater ${ }^{\circledR} 18$ na parte externa da orelha e injeção venosa de Tiopental sódico - 12,5 mg/ kg. A manutenção venosa foi obtida com solução fisiológica a 0,9\% na dose de $10 \mathrm{mg} / \mathrm{kg} / \mathrm{h}$.

Foi realizada anestesia com Tiopental sódico; intubação orotraqueal e ventilação mecânica com fração de inspiração de oxigênio de 40\%, e volume corrente de $10 \mathrm{ml} / \mathrm{kg}$; intubação orotraqueal guiada por câmera Rüsch ${ }^{8} 7.0$, insuflação do balonete com $3 \mathrm{ml}$ de ar e ventilação mecânica em respirador Takaoka 670 com $\mathrm{FiO} 2$ de $40 \%$, com freqüência respiratória de 12 incursões por minuto e volume corrente de $10 \mathrm{ml} / \mathrm{kg}$. Desta maneira foi possível obter os valores da pressão endotraqueal.

A manutenção anestésica foi obtida com halotano inalatório e injeção endovenosa de Pancurônio na dose de $0,8 \mathrm{mg} / \mathrm{kg}$ a cada 30 minutos.

Após a fixação do animal à mesa operatória, foi realizada cervicotomia direita para cateterização da veia jugular externa direita com cateter de Swan-Ganz ${ }^{\circledR}$ BAXTER 7F, após ligadura distal com fio de algodão 00, para determinação das pressões do átrio direito, do tronco da artéria pulmonar, do capilar pulmonar e coleta de sangue venoso misto, no tronco da artéria pulmonar.

Pelo mesmo acesso, foi introduzido cateter de polietileno $8 \mathrm{~F}$ na extremidade distal da artéria carótida comum direita, para registro contínuo da pressão arterial sistêmica e coleta de amostras arteriais.

Após incisão de pele de dois centímetros à esquerda da cicatriz umbilical, introduziu-se agulha de Veress na cavidade abdominal por punção, para realização do pneumoperitônio, de modo que a pressão intra-abdominal não ultrapassasse $16 \mathrm{mmHg}$.

Esse procedimento foi realizado nos três grupos experimentais, após o qual foram obtidas amostras gasométricas para ajuste de freqüência respiratória e volume corrente do respirador.

\section{Modelo de lesão diafragmática}

Após o pneumoperitônio, com pressão intra-abdominal aproximada de $15 \mathrm{mmHg}$, foi retirada a agulha de Verres e introduzido, na mesma incisão, trocarte de $10 \mathrm{~mm}$ da marca Auto-Suture ${ }^{\circledR}$, o qual deu passagem à óptica.

Realizado o inventário da cavidade abdominal, identificado o centro tendíneo do diafragma sendo então introduzidos, sob visão direta, dois trocatres de $10 \mathrm{~mm}$ e $5 \mathrm{~mm}$ nos hipocôndrios direito e esquerdo, para introdução de porta-agulhas e pinça de apreensão. Em todos os animais, foi introduzido, através do trocarte de $10 \mathrm{~mm}$, um fio de marcapasso monycron $20 \mathrm{com}$ agulha de $2,5 \mathrm{~cm}$, de modo que toda a sua extensão permanecesse na cavidade abdominal. Após este procedimento, foi realizado o sorteio.

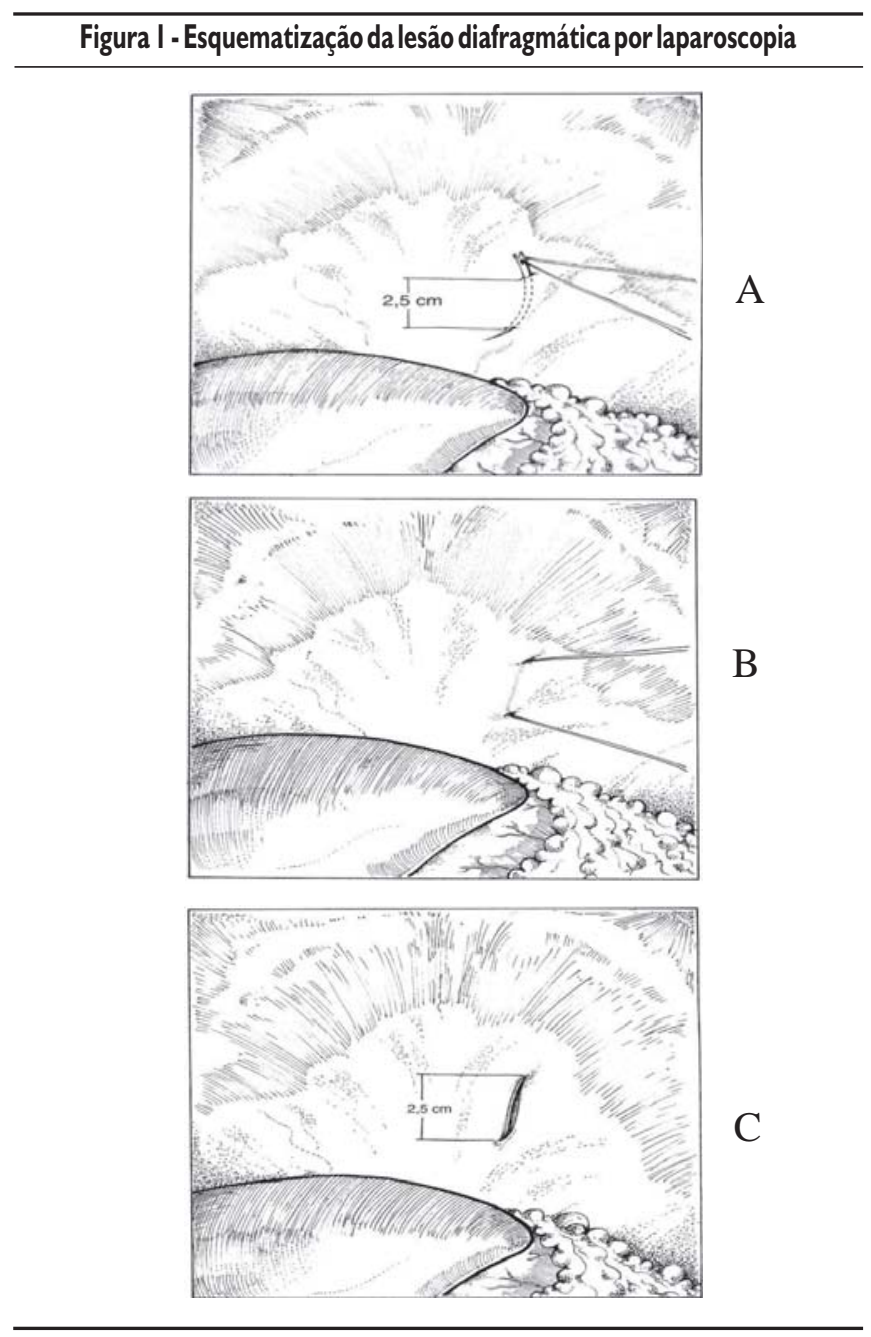

Legenda - Passagem da agulha no centro tendíneo do diafragma no sentido ântero- posterior (A); Exteriorização das pontas do fio de marcapasso (B); Tração das pontas do fio por movimento em "serra", a fim de provocar a lesão diafragmática (C)

Quadro I - Esquematização das fases experimentais (FI, F2, F3, F4, F5, F6)

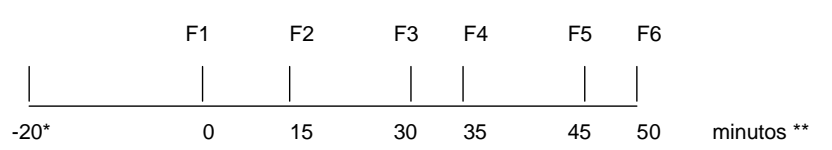

Preparo do modelo $(*)$

Sorteio / Lesão diagragmática (Grupos I e 3)(*)

FI - Pneumoperitônio $15 \mathrm{mmHg}$ (Grupos 2 e 3) / coleta de dados

F2 - Coleta de dados

F3 - Radiografia de tórax. Esvaziamento pneumoperitônio (Grupos 2e 3).

F4 - Coleta de dados

F5 - Pneumoperitônio com $25 \mathrm{mmHg}$ (grupos I, 2 e 3) / coleta de dados

F6 - coleta de dados/morte dos animais

(*) A - Indução anestésica / Anestesia; B - Acesso vascular; C - Pneumoperitônio, Fio de marca passo na cavidade abdominal, Sorteio; D - Drenagem do pneumoperitônio; E - Lesão (grupos I e 3) /

Pneumoperitônio (grupos 2 e 3)

(**) Morte dos animais 


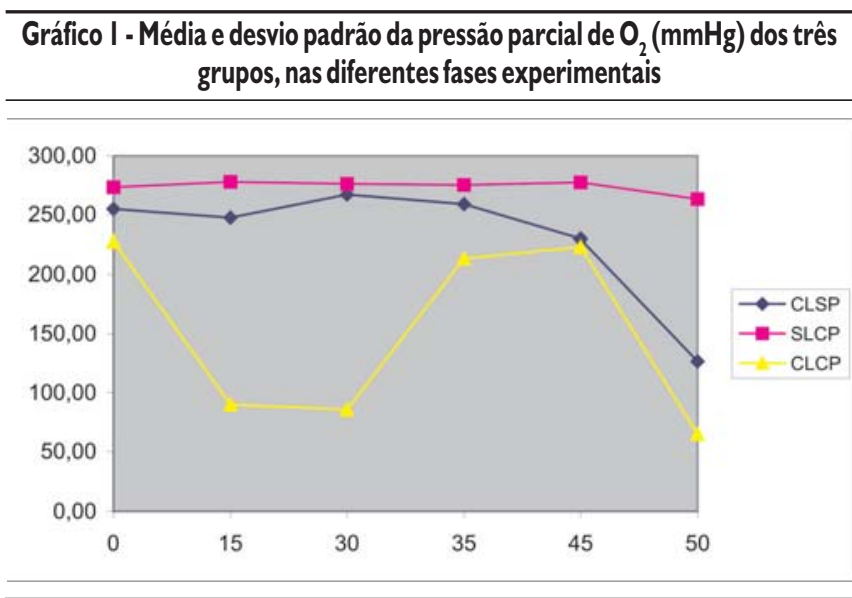

\section{Gráfico 2-Média e desvio padrão da saturação de 02 no sangue arterial} $(\mathrm{mmHg})$ dos três grupos, nas diferentes fases experimentais

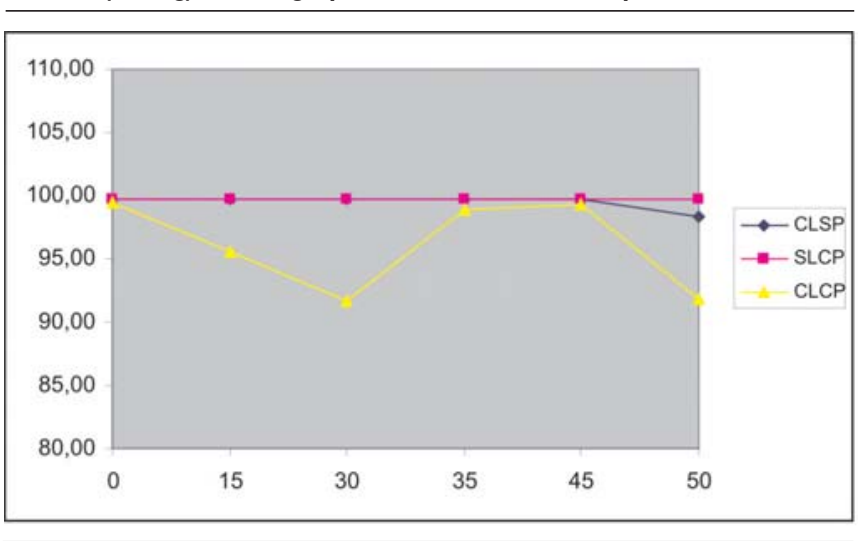

Nos animais dos grupos I e 3, a lesão diafragmática foi realizada através de passagem da agulha no centro tendíneo do diafragma no sentido ântero-posterior e exteriorização de suas pontas na cavidade abdominal (Figura I-A).

As duas pontas do fio foram então tracionadas delicadamente pelo porta-agulhas através do trocarte de $10 \mathrm{~mm}$, o qual foi retirado, sendo então exteriorizadas através da parede abdominal, tomandose a precaução de não tracioná-las para que não houvesse laceração diafragmática (Figura I-B) .

Nos animais do grupo 2 (SLCP), o fio permaneceu solto na cavidade, sendo retirado apenas o trocarte de $5 \mathrm{~mm}$, o que permitiu o esvaziamento do pneumoperitônio. Nos animais do grupo I e 3 , as pontas do fio foram tracionadas por movimento em "serra", a fim de provocar a lesão no diafragma (Figural-C).

\section{Fases da experimentação}

Em todos os grupos estabeleceu-se um período de 20 minutos entre a conclusão do procedimento laparoscópico e o início da avaliação, a qual teve a duração de 50 minutos, dividida em seis fases, para registro dos parâmetros respiratórios e hemodinâmicos. (Quadro I). Nota-se que as medidas foram

\section{Gráfico 3 - Média e desvio padrão da saturação de 02 no sangue venoso misto} $(\mathrm{mmHg})$ dos três grupos, nas diferentes fases experimentais

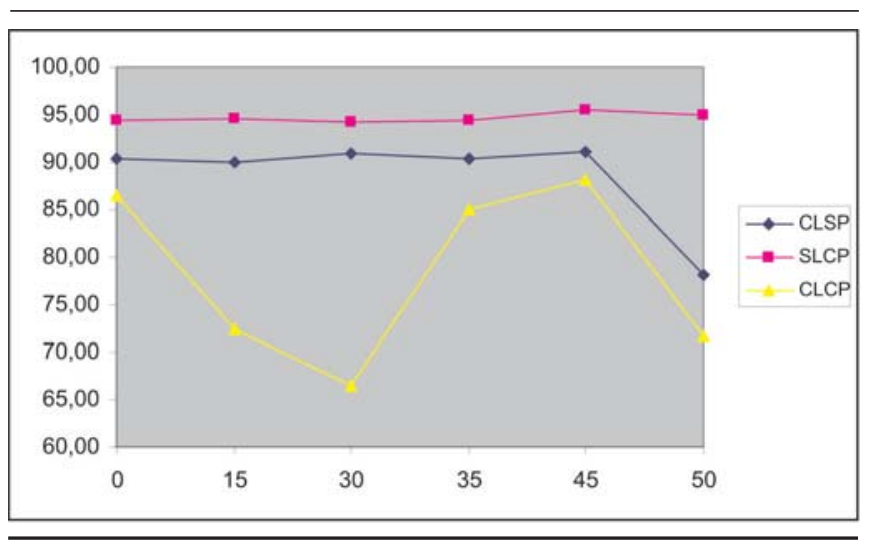

Gráfico 4 - Média e desvio padrão da pressão média de artéria pulmonar $(\mathrm{mmHg})$ dos três grupos, nas diferentes fases experimentais

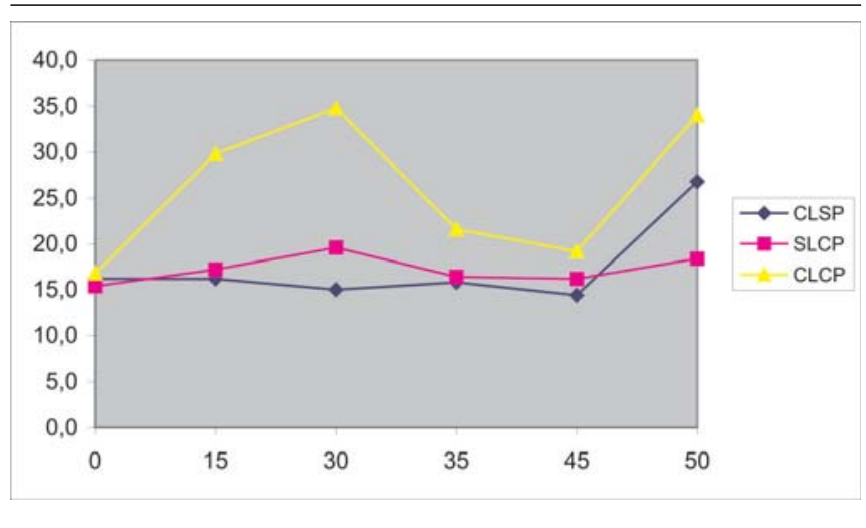

tomadas na vigência do pneumoperitônio com pressão de 15 $\mathrm{mmHg}$ e, ao final do experimento, com pressão de $25 \mathrm{mmHg}$.

Após a conclusão do experimento, os animais foram mortos com injeção endovenosa de cloreto de potássio a 19,1\%.

As variáveis analisadas foram:

Respiratórias: gasometrias arterial e venosa e pressão endotraqueal (PET).

Hemodinâmicas: freqüência cardíaca (FC), débito cardíaco (DC), pressão arterial média (PAM), pressão média de artéria pulmonar (PAP), pressão de capilar pulmonar (PCP), índice cardíaco (IC).

\section{Análise estatística}

Os grupos foram comparados entre si em cada uma das fases através de uma Análise de Variância (ANOVA). Quando detectadas diferenças significativas, utilizou-se o Teste de Comparações Múltiplas de Scheffé para localizar estas diferenças.

Para cada grupo foram comparadas as fases através da aplicação de uma Análise de Variância com medidas repetidas. Adotou-se como nível de significância o valor 0,05 (5\%). Com isso, níveis descritivos ( $p$ ) inferiores a esse foram considerados significativos $(p<0,05)$. 


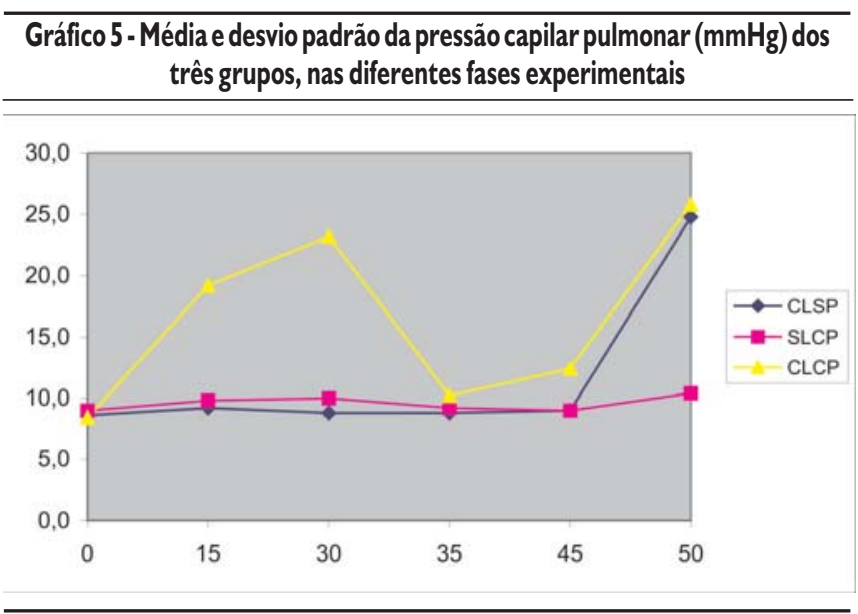

\section{Gráfico 6 - Média e desvio padrão do débito cardíaco (L/min) dos três grupos, nas diferentes fases experimentais}

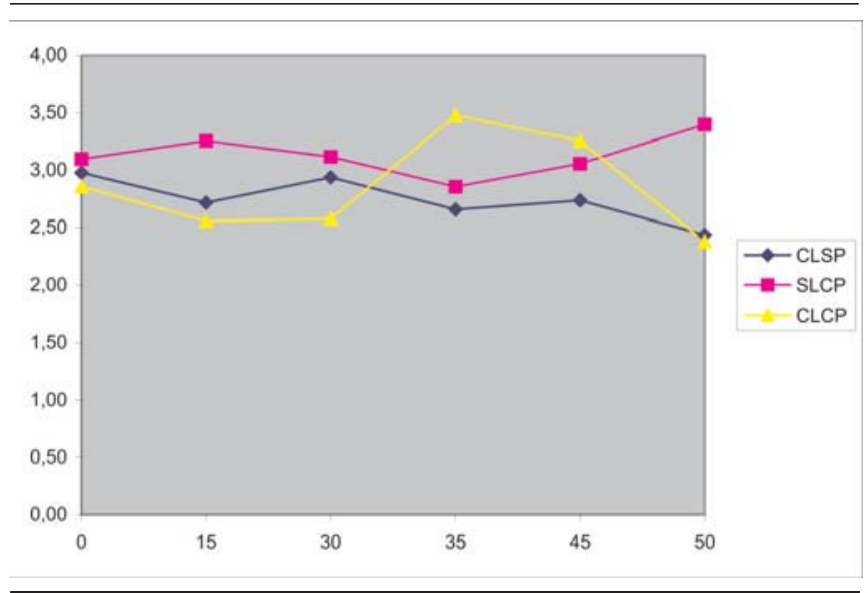

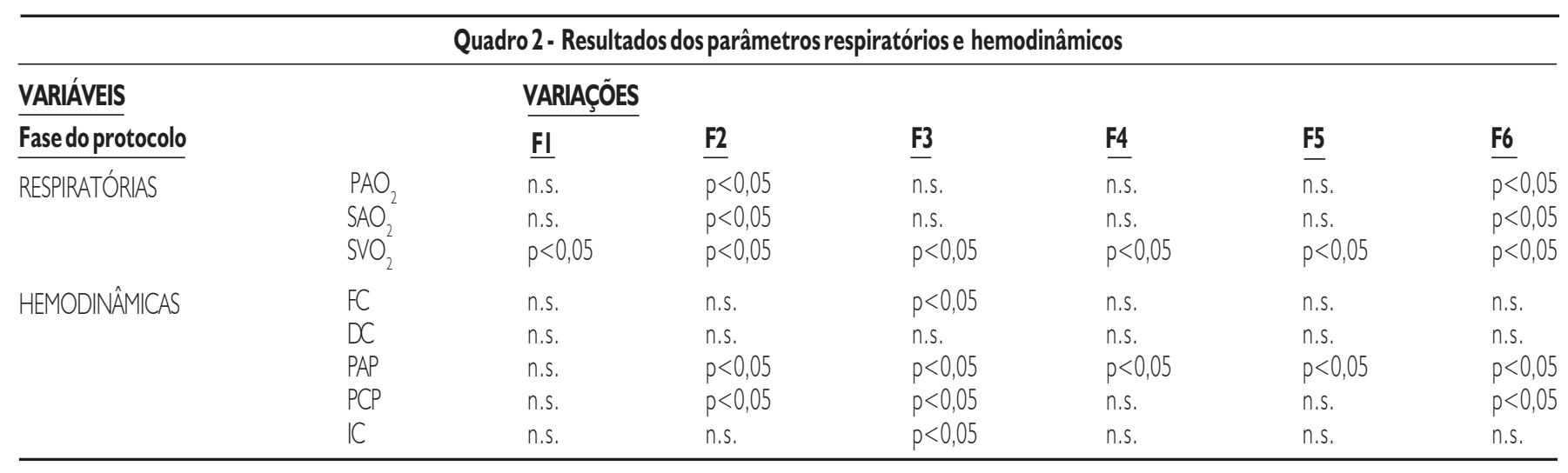

\section{Resultados}

Com relação às variáveis respiratórias houve alterações significativas na $\mathrm{PAO}_{2}$ (Gráfico I), $\mathrm{SAO}_{2}$ (Gráfico 2 ) e $\mathrm{SVO}_{2}$ (Gráfico 3).

Com relação às variáveis hemodinâmicas houve alterações significativas na pressão de artéria pulmonar (PAP) (Gráfico 4) e pressão de capilar pulmonar (PCP) (Gráfico 5) .

Não houve alterações no débito cardíaco (Gráfico 6).

\section{Discussão}

A idealização do modelo de lesão diafragmática foi caracterizada pela dificuldade em reproduzir situação semelhante à que ocorre nos ferimentos da transição tóraco- abdominal por arma branca ${ }^{25-26}$. Existe uma publicação que demonstra lesão experimental realizada por meio de toracotomia, com acesso ao diafragma por visão direta, mas que não utiliza o pneumoperitôni ${ }^{27}$.

Optou-se por se realizar o experimento utilizando o pneumoperitônio com pressões de $15 \mathrm{mmHg}$ e $25 \mathrm{mmHg}$, com o objetivo de averiguar se o cirurgião pode utilizar com segurança uma pressão mais elevada que a habitualmente utilizada na prática clínica.

Houve alteração significante na pressão da artéria pulmonar, especialmente no grupo 3 (CLCP), mesmo quando já não havia pneumoperitônio, sendo que nos grupos I (CLSP) e 2 (SLCP) tiveram comportamento semelhante entre si, o que mostra que $\mathrm{O}$ fator mecânico (pneumoperitônio) não deve exercer papel fundamental nesse aumento, e sim a presença de um eventual pneumotórax associado à lesão do diafragma.

As alterações da pressão de capilar pulmonar nos 30 minutos iniciais foram significantes no grupo 3 (CLCP). Entre os grupos I (CLSP) e 2 (SLCP) houve comportamento semelhante, o que pode ser justificado pelos efeitos de um possível pneumotórax.

O aumento da pressão abdominal pelo pneumoperitônio e a lesão do diafragma, que condicionou a possibilidade de pneumotórax, não causaram repercussão hemodinâmica, exceto quando se utilizou pressão de $25 \mathrm{mmHg}$, que determinou alteração em todas as variáveis estudadas.

Houve um decréscimo significativo da $\mathrm{PAO}_{2}$ nos animais submetidos à lesão do diafragma e pneumoperitônio (CLCP) em relação aos demais, durante os 30 minutos iniciais, apesar de não ter havido hipóxia. Não há explicação aparente para essa diminuição, pois o débito cardíaco esteve constante. A saturação de oxigênio, nessas fases, apresentou diferença significante, com diminuição no grupo 3 (CLCP) em relação aos outros sem, no 
entanto, ter atingido um valor incompatível com a normalidade.

A interpretação dos resultados deste estudo baseou-se em dois aspectos: um, decorrente do pneumoperitônio com aumento da pressão intra-abdominal e elevação do diafragma; o outro, a comunicação entre as cavidades torácica e abdominal, com formação de pneumotórax.

A avaliação dos resultados mostra que o pneumoperitônio isolado não determina alterações hemodinâmicas e respiratórias, que só ocorrem quando ele está associado à lesão do diafragma.

Soldá, em 1993, ao analisar o emprego da laparoscopia diagnóstica no trauma em 60 doentes, não observou pneumotórax nos portadores de lesão do diafragma, o que talvez possa ser explicado pelo gradiente de pressão existente entre as cavidades torácica e abdominal, que determina o "tamponamento" do orifício diafragmático por epíplon. Mesmo após a redução do epíplon para o abdome, não houve pneumotórax, possivelmente devido à pressão positiva determinada pelo respirador ${ }^{33}$.

Em estudo experimental, Perlingeiro, em 200I, destacou que a obliteração do orifício por epíplon pode ser um dos fatores que eventualmente possam interferir na cicatrização de uma lesão diafragmática ${ }^{34}$.

Apesar da contribuiç̧ão significativa no diagnóstico de afecções abdominais na urgência, a videolaparoscopia é um método invasivo, que requer anestesia geral, não é um procedimento isento de riscos e uma falha na sua indicação ou realização pode ser grave para o traumatizado ${ }^{35-37}$.

\section{CONCLUSÃO}

A videolaparoscopia tem sido utilizada de forma sistemática nos ferimentos por arma branca da transição tóraco-abdominal, em doentes assintomáticos, ou seja, sem indicação de toracotomia ou laparotomia.

Por este motivo, acreditamos que este estudo possa corroborar o seu valor como método seguro na avaliação de doentes portadores de lesão do diafragma, desde que obedecidos os princípios técnicos, hoje muito bem definidos.

\section{Conflito de interesse: não há.}

\section{SUMMARY}

\section{Hemodynamic and Respiratory CHANGES CAUSED by CO2} LAPAROSCOPY IN AN EXPERIMENTAL MODEL OF DIAPHRAGMATIC INJURY

OBJECTIVE. The objective of this study was to analyze the hemodynamic and respiratory changes caused by $\mathrm{CO}_{2}$ laparoscopy in an experimental model of diaphragmatic injury.

METHODS. Fifteen animals chosen at random were submitted to diaphragmatic injury by means of $\mathrm{CO}_{2}$ laparoscopy. Evaluation consisted of drawing blood samples to analyze blood gases, as well as the hemodynamic and respiratory variables.

RESULTS: Alterations occurred in APA, CPP, PAO $, \mathrm{ASO}_{2}, \mathrm{VSO}_{2}$.

CONCLUSION: We concluded that videolaparoscopy with pneumoperitoneum pressure under $15 \mathrm{mmHg}$ is a safe procedure in patients with diaphragmatic injuries because this pressure level does not cause hemodynamic changes, such as decrease of the cardiac output. [Rev Assoc Med Bras 2007; 53(4): 294-9] KEY WORDS: Diaphragm. Laparoscopy. Pneumoperitoneum.

\section{REFERÊNCIAS}

I. Ivatury RR, Simon RJ, Weksler B, Bayard V, Stahl WM. Laparoscopy in the evaluation of the intrathoracic abdomen after penetrating injury. I Trauma. 992;33:101-9.

2. Shaftan GW. Indications for operation in abdominal trauma. Am J Surg. 1960;99:657.

3. Moore JB, Moore EE, Thompson JS. Abdominal injuries associated with penetrating trauma in the lower chest. Am J Surg. I980; I 40:724-30.

4. Payne HJ, Yellin A E. Traumatic diaphragmatic hernia. Arch Surg. 1982; |17:18-24.

5. Hirshberg A, Thomson SR, Bade PG, Huizinga WKJ. Pitfalls in the management of penetrating chest trauma. Am J Surg. 1989; | 57:372-5.

6. Feliciano DV, Cruse PA, Mattox KL, Bitondo CG, Burch JM, Noon GP, Beall AC. Delayed diagnosis of injuries to the diaphragm after penetrating wounds. J Trauma. 1988;28: I 135-44.

7. Meyers BF, McCABE CJ. Traumatic diaphragmatic hernia. Occult marker of serious injury. Ann Surg. 1994;218:783-90.

8. Kligman M, Saxe J, Hai SA.Delayed detection of a traumatic diaphragmatic injury caused by positive pressure ventilation: a case report and a proposed system of a classification of delayed diagnosis of traumatic injuries. Int Surg. 2005;90:125-9.

9. Rasslan S, Soldá SC, Rodrigues FCM. Análise crítica da vídeolaparoscopia na urgência abdominal não traumática. In: Margarido NF, Saad Junior R, Cecconello I, Martins JL, Paula RA, Soares LA. Vídeocirurgia: Colégio Brasileiro de Cirurgiões. Capítulo de São Paulo. São Paulo: Robe; 1994. p.7I-85.

10. Clay RC, Hanlon CR. Pneumoperitoneum in the differential diagnosis of diaphagmatic hernia. J Thorac Surg. 1951;20:57-69.

I I. Freeman T, Fischer RP. The inadequacy of peritoneal lavage in diagnosing acute diaphragmatic rupture. J Trauma. 1976; 16:538-42.

12. Thal ER. Evaluation of peritoneal lavage and local exploration in lower chest and abdominal stab wounds. J Trauma. 1977;17:642.

1 3. Thompson JS, Moore EE. Peritoneal lavage in the evaluation of penetrating abdominal trauma. Surg Gynecol Obstet. 1981;153:861-3.

I 4. Shea LS, Graham AD, Fletcher JC. Diaphragmatic injury: a method of early diagnosis. J Trauma. 1982;22: 539-43.

I5. Merlotti G], Dillon BC, Lange DA, Robin AP, Barrett JA. Peritoneal lavage in penetrating thoraco-abdominal trauma. J Trauma. 1985;28:17-23.

16. Frantzides CT, Carlson MA. Laparoscopic repair of a penetrating injury to the diaphragm: a case report. J. Laparoendosc Surg. 1994;4:I53-6.

17. Murray JÁ, Demetriades D, Asensio JÁ, Cornwell EE 3RD, Velmahos GC, Belzberg $H$, et al. Occult injuries to the diaphragm: prospective evaluation of laparoscopy in penetrating injuries to the left lower chest, J Am Coll Surg. 1998; 187:626-30.

I 8. Macquay N Jr., Britt LD. Laparoscopy in the evaluation of penetrating thoracoabdominal trauma. Am Surg. 2003;69:788-91.

19. Mahajna A, Mitkal S, Bahuth H, Krauz MM. Diagnostic laparoscopy of penetrating injuries in the thoracoabdominal region. Surg Endosc. 2004; | 8: | 485-7.

20. Albert VM, Mamel JJ. Delayed tension pneumothorax after laparoscopy. Surg Laparosc Endosc. 1997;7:70-2.

21. Wiedeman JE, Knolmayer TJ, Bowyer MW. Is tension pneumothorax: a threat in trauma laparoscopy? JTrauma. 1998;45:677-83.

22. Shiraki K, Hamada M, Sugim'oto K, Ito T, Murata K, Fujikawa K, et al. Pneumothorax after diagnostic laparoscopy. Hepatogastroenterology. 2002;49: I033-5.

23. Ludemann R, Krysztopik R, Jamieson GG, Watson DI. Pneumothorax during laparoscopy. Surg Endosc. 2003;17:1985-9. 
24. Leighton TA, Bongard FS. Comparative cardiopulmonary effects of helium and carbon dioxide pneumoperitoneum. Surg Forum. 1991:50:485-7.

25. Bautz PC, Knottnbelt JC. Diaphragmatic injury: a source of hazard in laparoscopy. Injury. 1994;25:40 I-2.

26. Thomas P, Moutardier V, Ragni J, Giudicelli R, Fuentes P. Video-assisted repair of a ruptured right hemidiaphragm. Eur Cardiothorac Surg. 1994;8:157-9.

27. Ali J, Qi W. The cardiorespiratory effects of increased intra-abdominal pressure in diaphragmatic rupture. J Trauma. 1992;33:233-9.

28. Smith I, Benzie RJ, Gordon NLM, Kelman GR, Swapp GH. Cardiovascular effects of peritoneal insufflation of carbon dioxide for laparoscopy. Brit Med J. 1971;14:410-I.

29. Marshall RL, JEBSON PR, Davie IT. Circulatory effects of carbon dioxide insufflation of the peritoneal cavity for laparoscopy. $\mathrm{Br} J$ Anaesth. 1972:4:680-4.

30. Cameron E, Pocock J, Dear GL. Gas exchange in the abdominal cavity during laparoscopy. I R Soc Med. 1983;76:1015-8.

3 I. Livingston DH, Tortella BJ, Blackwood J, Machiedo GW, Rush B F. The role of laparoscopy in abdominal trauma. J Trauma. 1992;33:47|-5.

32. Benhamou D, Simonneau G, Poynard T, Goldman M, Chaput JC, Duroux $P$. Diaphragm function is not impaired by pneumoperitoneum after laparoscopy. Arch Surg. 1993; 128:430-2.

33. Soldá SC. Laparoscopia diagnóstica no trauma abdominal [dissertação]. São Paulo: Faculdade de Ciências Médicas da Santa Casa de São Paulo; 1993.
34. Perlingeiro JAG. História natural do ferimento diafragmático produzido por agente perfuro cortante. Estudo experimental em ratos [tese]. São Paulo: Faculdade de Ciências Médicas da Santa Casa de São Paulo, 2001.

35. Salvino CK, Esposito TJ, Marshall WJ, Dries DJ, Morris RC, Gamelli RL. The role of diagnostic laparoscopy in trauma patients. J Trauma. 1993;34:506-I 5.

36. Rossi P. Mullins D. Thal E. Role of laparoscopy in evaluation of abdominal trauma. Am J Surg. 1993;166:707-11.

37. Leppaniemi A, Haapiainen R. Occult diaphragmatic injury caused by stab wounds. J Trauma. 2003;55:646-50.
Artigo recebido: 18/05/06

Aceito para publicação: 09/05/07 